\title{
Elementos del contexto que intervienen en el desarrollo de las políticas públicas de salud sexual y salud reproductiva elaboradas entre el 2003 y el $2013^{*}$
}

\section{Contextual Elements Involved in the Development of Sexual and Reproductive Health Public Policies Made Between 2003 and 2013}

\section{Elementos do contexto que intervêm no desenvolvimento das políticas públicas de saúde sexual e saúde reprodutiva elaboradas entre o ano 2003 e o 2013}

Fecha de recepción: 28-01-15 Fecha de aceptación: 27-04-15 Disponible en línea: 30-06-15

doi: 10.11144/Javeriana.rgyps18-28.ecid

Como citar este artículo:

Rodríguez-Vargas FA, Molina-Berrio DP. Elementos del contexto que intervienen en el desarrollo de las políticas públicas de salud sexual y salud reproductiva elaboradas entre el 2003 y el 2013. Rev. Gerenc.

Polít. Salud. 2015; 14(28): 10-30. http://dx.doi.org/10.11144/Javeriana.rgyps18-28.ecid

Fainory Andrea Rodríguez-Vargas**

Diana Patricia Molina-Berrio***

Este artículo es resultado de la investigación sobre el estado del arte de las políticas públicas en salud sexual y salud reproductiva en los municipios del área metropolitana del Valle de Aburrá, 2003-2013.

** Administradora en Salud: Gestión de Servicios de Salud, investigadora de la Universidad de Antioquia. Dirección postal: Universidad de Antioquia, Facultad de Medicina, Carrera 51d № 62-29, Bloque Manuel Uribe Ángel Oficina 202, Tel: 2196071.Correo electrónico: fainory.rodriguez@udea.edu.co.

*** Magíster en Salud Pública, psicóloga, docente e investigadora de la Universidad de Antioquia. 


\section{Resumen}

Objetivo: describir los elementos del contexto que intervienen en el desarrollo de las políticas públicas en salud sexual y reproductiva de los municipios del área metropolitana. Metodología: se realizó una revisión sistemática de políticas públicas, publicaciones científicas y entrevistas a expertos en temas de salud sexual y reproductiva. El análisis de la información se hizo mediante el proceso de codificación y categorización. Resultados: los hallazgos muestran que la sSR es un tema que ya se discute en los diferentes grupos sociales y convoca la opinión pública; ha tenido cambios de enfoques de tipo social y cultural, se reconoce a los actores y la relación entre políticas públicas y medios de comunicación como transformadores de la ciudadanía. La política nacional de SSR es pauta para los entes municipales, pero sus lineamientos se deben adaptar a las necesidades particulares de los municipios, con el fin de mejorar la prestación del servicio según el contexto de sus habitantes.

Palabras clave: políticas; política de salud; salud sexual y reproductiva; derechos sexuales y reproductivos; salud sexual; salud reproductiva

\section{Abstract}

Objective: Describing the contextual elements involved in the development of sexual and reproductive health public policies of the municipalities of the metropolitan area. Methodology: We performed a systematic review of the public policies, scientific journals, and interviews to experts on sexual and reproductive health topics. The analysis of the information was performed through a codification and categorization process. Results: The findings show that SRH is a topic being discussed in different social groups and brings about public opinion. It has undergone social and cultural changes in approach, there is recognition of the actors and the relationship between public policies and the media as elements of transformation of citizens. The national policies of SRH are a pattern to follow for municipal entities, but their guidelines must adapt to the particular necessities of each municipality as to improve the rendering of the services according to the context of the inhabitants.

Keywords: policies; health policies; sexual and reproductive health; sexual and reproductive rights; sexual health; reproductive health

\section{Resumo}

Objetivo: descrever os elementos do contexto que intervêm no desenvolvimento das políticas públicas em saúde sexual e reprodutiva dos municípios da área metropolitana. Metodologia: foi realizada uma revisão sistemática de políticas públicas, publicações científicas e entrevistas a peritos em temas de saúde sexual e reprodutiva. A análise da informação foi feita mediante processo de codificação e categorização. Resultados: os achados mostram que a sSR é um tema já discutido nos diferentes grupos sociais e convoca a opinião pública; tem tido mudanças de enfoque de tipo social e cultural, reconhece-se aos atores e o relacionamento entre políticas públicas e mídia como transformadores da cidadania. A política nacional de ssR é pauta para os entes municipais, mas as diretrizes ainda têm de se adaptar às necessidades particulares dos municípios, com o fim de melhorar a prestação do serviço de acordo ao contexto dos habitantes.

Palavras-chave: políticas; política de saúde; saúde sexual e reprodutiva; direitos sexuais e reprodutivos; saúde sexual; saúde reprodutiva 


\section{Introducción}

La salud sexual y la salud reproductiva han constituido un tema central en las agendas políticas nacionales e internacionales. Desde la Conferencia de Derechos Humanos realizada en Teherán en 1968, se reconoció el derecho a la reproducción por parte de los padres. La Conferencia sobre Población (Bucarest, 1974) amplió este alcance al "derecho de las parejas e individuos", y la conferencia realizada en ocasión del Año Internacional de la Mujer (México, 1975) abordó el tema de la reproducción desde la perspectiva del "derecho a la integridad corporal y al control de las mujeres sobre su capacidad reproductiva”. La Conferencia sobre Población y Desarrollo (El Cairo, 1994) ratificó el derecho de las mujeres a controlar su propia fecundidad a través del acceso y la información sobre los métodos de anticonceptivos, mientras que el Programa de Acción propuesto en esta conferencia apuntó a promover la equidad y la igualdad entre los sexos, los derechos de la mujer y la eliminación de la violencia hacia esta (1).

En 1994, en la Conferencia de Población y Desarrollo celebrada en El Cairo, se asumió un concepto más positivo de la sexualidad y la reproducción, reconociendo a las relaciones personales, la educación y el acceso a los servicios como factores que influyen en su efectivo desarrollo (2). Por su parte, la Plataforma de Acción Mundial aprobada en la IV Conferencia Mundial sobre la Mujer (Beijing, 1995) reafirmó y fortaleció los avances logrados en El Cairo. Se sugirió además que los gobiernos aseguraran la igualdad y la no discriminación de las mujeres adoptando leyes y medidas para proteger sus derechos. Finalmente, ese mismo año la Federación Internacional de Planificación Familiar (IPPF) aprobó una Carta de Derechos Sexuales

12 y Reproductivos en la que reconoce doce derechos como instrumentos legales inter- nacionales y los posiciona al mismo nivel de los Derechos Humanos, solicitando que sean considerados en cartas, convenciones y pactos de las Naciones Unidas (1).

Para el año 2000 la Conferencia del Milenio amplió más la comprensión de este concepto, ubicando la salud sexual y reproductiva (SSR) como un indicador de desarrollo de las naciones. Dicho indicador habría de medirse en aspectos como la salud materna, la equidad entre los géneros y la disminución del VIH/Sida y otras infecciones sexualmente transmisibles (3). La Organización Mundial de la Salud (oms), por su parte, aportó al desarrollo del concepto incorporando el tema a la investigación en las ciencias sociales y humanas, lo cual obligó a darle una nueva visión, perspectiva e interacción, desde diferentes enfoques, aplicando una mirada más compleja al campo de la reproducción y la sexualidad de los seres humanos (4).

Esta concepción integral de la SSR transformó la percepción del tema en el contexto cultural y normativo, acogiendo los derechos sexuales y reproductivos (DSR) y ampliando el interés por brindar a los sujetos la posibilidad de cubrir aspectos que les permitan regular y tener control autónomo y responsable sobre todas las cuestiones relativas a la sexualidad, sin ningún tipo de coacción, violencia, discriminación, enfermedad o dolencia (4). Los DSR constituyen el soporte básico en los diferentes temas de sSR, por su relación con la autodeterminación corporal y sexual, asociada a los derechos humanos fundamentales que aspiran a ser garantizados por el Estado mediante la ejecución de las políticas y que son considerados de cumplimiento con carácter universal (4).

Las condiciones para el ejercicio de los derechos humanos relativos a la SSR en la población se encuentran ligadas a la implementación de las políticas públicas, permi- 
tiendo reflejar el nivel de desarrollo y las inequidades que afectan a las comunidades; también se relacionan con las posibilidades de acceso y carencia en atención de los servicios de salud, con el nivel de educación e información, los índices de pobreza y en general con las condiciones socioculturales de la población (5).

Las actividades que desarrollan los entes territoriales nacionales y municipales en temas de ssR, definidos por ellos como prioritarios, buscan brindar a personas, familias y comunidades, respuestas y oportunidades para el ejercicio efectivo de los DSR, tal como se encuentra establecido en la Política Nacional de Salud Sexual y Salud Reproductiva del Ministerio de la Protección Social del año 2003. No obstante, estas han sido pocas o han estado marcadas por intereses particulares y no por el abordaje social que se le debería dar al desarrollo de los proyectos, planes, programas y estrategias que se derivan para la ejecución de las políticas públicas (6).

Lo anterior trae como consecuencia altibajos en los indicadores de medición de los temas de SSR que se formularon con la política nacional. Estos fueron trasladados a los municipios del área metropolitana del Valle de Aburrá y en el 2011 se presentaron unos rangos altos en algunos de ellos: el 99,26\% de los partos atendidos contaron con por personal calificado; el porcentaje de nacidos vivos con cuatro o más controles prenatales fue de $87,89 \%$; la proporción de mujeres que fallecieron a causa de complicaciones relacionadas con el embarazo o el parto fue de 36,97 por cada 100000 nacidos; la tasa de mortalidad por cáncer de cuello uterino fue de 5,82 por cada 100000 mujeres; el $3,09 \%$ de las mujeres presentan maltrato físico, psicológico o sexual por parte de su pareja, en tanto que la relación de mujeres adolescentes embarazadas fue de 0,98 , en el rango entre quince y diecinueve años de edad, en comparación con el total de mujeres del mismo rango de edad (7).

Todos estos indicadores aún se encuentran en niveles bajos con respecto a las metas establecidas en los Objetivos del Desarrollo del Milenio (ODM) para Colombia, base de la política nacional, y no han permitido cumplir las metas establecidas en los planes de desarrollo y las políticas públicas existentes en los municipios en el tema de ssR (8). A partir de esto, los planes de desarrollo y las políticas públicas nacen para tratar de dar respuesta a problemas propios de las comunidades, traducidos en forma de normas, instituciones, prestaciones, bienes públicos o servicios, en donde cada municipio debía enfrentar de manera prioritaria aquellos lineamientos que se consideraran de mayor incidencia.

Desde la teoría, las políticas públicas son un conjunto de acciones y decisiones encaminadas a solucionar problemas propios de las comunidades, a través de normas, instituciones, prestaciones, bienes públicos o servicios. Es por ello que además de los documentos normativos, para el análisis se tuvieron en cuenta los planes de desarrollo, ya que en los municipios a estos se les considera políticas públicas y son los documentos que permiten llevar al terreno las actividades en el tema de ssR (9).

Como bien lo expresa Roth Dubel, las políticas públicas, su proceso de formación y su desarrollo tienen una complejidad mayor que cualquier teoría o modelo analítico que pretenda explicarlas (10). El abordaje de este objeto de estudio, desde cualquier perspectiva epistemológica o metodológica, tendrá como alcance dar "una mirada", una posibilidad de comprensión, que en este caso se espera sea valiosa para su análisis y para la toma de decisiones políticas. Considerando lo anterior, el presente estudio pretende realizar una descripción del escenario donde se 
materializan las políticas públicas en SSR en el contexto del área metropolitana. Asimismo, hace una lectura analítica de cómo algunos elementos identificados como preponderantes en el desarrollo de la política influyen en su desarrollo.

La lectura de estos elementos se realizó desde la perspectiva de la Teoría Crítica, de acuerdo con la cual se asume que la comprensión de la realidad se ve influida por la subjetividad de quienes realizan el ejercicio de comprensión. En este sentido, la objetividad se considera una ilusión. El investigador tiene una apuesta política que no teme reconocer y asumir, y esta apuesta se dirige a la transformación de una realidad que considera problemática para algunos grupos sociales. El ejercicio investigativo tiene sentido en tanto posibilita la emancipación, señala las formas de dominación y propone maneras de transformarlas (10).

Fue por ello que se pretendió analizar los documentos que implementan los municipios considerados, como políticas, planes de desarrollo, artículos publicados, la opinión de expertos, así como una búsqueda desde su contenido estructural que permitiera hallar los elementos intervinientes de las políticas públicas de salud sexual y salud reproductiva en los municipios del área metropolitana del Valle de Aburrá entre el 2003 y el 2013, y esto cómo ha aportado a los cambios sociales que vive la sociedad colombiana en el abordaje de la sexualidad y la reproducción.

\section{Materiales y métodos}

Los resultados que se presentan son el resultado de una investigación cualitativa con enfoque hermenéutico y su diseño fue un estado del arte. Se analizó el contenido

14 de políticas y textos, así como discursos, y a partir de una interpretación literal del tema y según el análisis de la información encontrada, para las diferentes fuentes primarias y secundarias se tuvieron en cuenta criterios de inclusión y exclusión.

En la recolección de la información se utilizaron como instrumentos dos fichas guía: una para la lectura de las políticas públicas y otra para la lectura de los artículos de investigación. Asimismo, se realizaron entrevistas semiestructuradas a expertos. Todos los instrumentos de recolección de información fueron diseñados bajo la guía de un sistema categorial elaborado a partir de los objetivos específicos del estudio, y del cual se identificaron las categorías: a) políticas públicas en salud sexual y reproductiva; b) salud reproductiva; c) salud sexual; d) conceptos sobre sexualidad; e) enfoques de la SSR; f) contenidos de las políticas en SSR; g) estrategia de las políticas en SSR; h) objetivos de las políticas; i) temáticas de las políticas en SSR; j) población objetivo/objeto de las políticas en SSR; k) seguimiento de las políticas en SSR; 1) monitoreo de las políticas en SSR; m) evaluación de las políticas; n) responsables de las políticas en SSR; o) medios de difusión y p) normativa que permitió recolectar y analizar las fuentes primarias y secundarias.

Para la recolección de las fuentes primarias se realizó la entrevista semiestructurada a seis expertos, lo que permitió ampliar la comprensión del objeto de análisis. Estas entrevistas se realizaron a personas que por su experiencia humana y laboral hicieron aportes para comprender la diversidad teórica y conceptual sobre las diferentes temáticas en la salud sexual y la salud reproductiva, como el aborto, el embarazo adolescente, el VIH, la mortalidad materna y otros temas nacientes, como nuevas masculinidades, población lesbiana, gay, bisexual, transgénero e intersexuales (LGBTI) y erotismo (11). 
El tipo de muestreo que se utilizó para la selección de estos expertos claves fue el intencionado. Una estrategia de muestreo deliberado para el estudio, con base en las necesidades de la información detectadas. Los criterios de inclusión de expertos fueron: a) persona avalada por un título académico; b) con mínimo dos años de experiencia en un tema relacionado con SSR; c) vinculada a centros de educación superior (universidades), organizaciones no gubernamentales (ONG), entidades del Estado o privadas, o activistas sociales; y d) que su lugar de trabajo esté dentro del entorno local del Valle de Aburrá (11).

Se contactó a los expertos por medio del rastreo de entidades que podrían brindar información sobre el tema, así como algunos que por su trayectoria en los temas de SSR son reconocidos localmente. Los expertos contactados trabajan los temas de: a) salud sexual, b) maternidad, c) salud sexual adolescente d) LGTBI, e) salud sexual-viH. Para la recolección de las fuentes secundarias se realizó la revisión bibliográfica de artículos de investigación, con el fin de analizar y sintetizar el material publicado sobre el tema de políticas públicas en salud sexual y reproductiva en los municipios del área metropolitana del Valle de Aburrá, en investigaciones realizadas entre el 2003 y el 2013 inclusive. Se utilzaron las bases de datos bibliográficas de las colecciones electrónicas que se tienen en el sistema de bibliotecas de la Universidad de Antioquia. Los criterios de inclusión de artículos fueron: a) cuyo objeto de estudio sean las políticas públicas de ssR de algunos de los municipios del Valle de Aburrá; b) publicado en los últimos diez años; c) ubicados en bases de datos especializadas; d) en idioma español o inglés (12).

Se hizo un perfil de búsqueda de la investigación de las posibles relaciones sintácticas entre las palabras clave que se derivaron del proyecto: política, salud sexual, salud sexual en Antioquia, política pública en salud sexual. Las bases de datos elegidas previamente referidas a revistas de biomedicina y ciencias de la salud fueron ApaPsyc Net, PubMed, Ebsco, Science Citation Index, Scielo, Dialnet. Como último recurso se decidió buscar en Google Académico, en donde se obtuvieron finalmente dos artículos que se muestran en la tabla 1 , los cuales cumplían con todos los criterios de inclusión.

Otra fuente secundaria fue la revisión de políticas públicas en los municipios del área metropolitana. Se solicitó a las secretarías de salud de los municipios del Valle de Aburrá y a dos municipios cercanos, las políticas públicas, programas, proyectos y estrategias en materia de sSR en el ámbito local y regional que desde el 2003 rigen en el municipio y que se pusieron en vigencia a partir de la política nacional de salud sexual. Entre los criterios de inclusión de políticas públicas se tuvieron en cuenta: a) documentos establecidos como acuerdos por el Concejo en uno de los municipios pertenecientes al

Tabla 1. Selección de artículos

\begin{tabular}{|l|l|l|}
\hline Autor(es) & Título y subtítulo & Referencia bibliográfica \\
\hline $\begin{array}{l}\text { Alejandro Gómez Ló- } \\
\text { pez, Adriana Serrano }\end{array}$ & $\begin{array}{l}\text { Metrosalud una herramienta de política } \\
\text { pública y construcción de ciudadanía }\end{array}$ & $\begin{array}{l}\text { Revista CEs Salud Pública. } \\
\text { 2012; 3 (2): 296-309 }\end{array}$ \\
\hline $\begin{array}{l}\text { Rita Elena Almanza } \\
\text { Payares }\end{array}$ & $\begin{array}{l}\text { Política local en salud sexual y reproductiva } \\
\text { como respuesta a la situación de salud en } \\
\text { Medellín, 2008-2011 }\end{array}$ & $\begin{array}{l}\text { Rev Salud Pública de Me- } \\
\text { dellín. 2008; 3(1): 87-101 }\end{array}$ \\
\hline
\end{tabular}

Fuente: elaboración propia 
Tabla 2. Políticas púBlicas Revisadas

\begin{tabular}{|l|l|l|}
\hline Nombre & Política publica & Ámbito \\
\hline Nacional de $\mathbf{2 0 0 3}$ & Política nacional de salud sexual y reproductiva & Colombia \\
\hline Acuerdo $\mathbf{0 8}$ de 2011 & $\begin{array}{l}\text { Política pública para el reconocimiento de la diversidad sexual } \\
\text { y las identidades de género, y para la protección, el restableci- } \\
\text { miento, la atención y la garantía de derechos de las personas } \\
\text { lesbianas, gays, bisexuales, transgeneristas e intersexuales (LGBTI) }\end{array}$ & $\begin{array}{l}\text { Municipio de } \\
\text { Medellín }\end{array}$ \\
\hline Acuerdo 20 de 2011 & $\begin{array}{l}\text { Política pública para la prevención y atención de las violen- } \\
\text { cias sexuales que afectan a la ciudadanía, principalmente a } \\
\text { mujeres, niñas y niños }\end{array}$ & $\begin{array}{l}\text { Municipio de } \\
\text { Medellín }\end{array}$ \\
\hline
\end{tabular}

Fuente: elaboración propia

área metropolitana del Valle de Aburrá; b) política pública diseñada y aprobada por entes territoriales; c) con expresión jurídica en ley, ordenanza y/o acuerdo; d) elaborados en los últimos diez años; e) con posibilidad de acceso al documento de forma virtual o física. Se obtuvieron solo dos documentos locales que cumplían con los criterios de inclusión, como lo muestra la tabla 2.

Dentro del análisis de las políticas locales también se incluyeron los planes de desarrollo de los municipios, ya que la administración los considera política pública, porque deben ser aprobados por el Concejo municipal de cada municipio.

Después de haber recolectado toda la información, se hizo la transcripción de entrevistas, así como el análisis de las fuentes secundarias por medio de las fichas guía. Además, se codificó, seleccionando los aportes más relevantes para la investigación, y se conformaron las categorías emergentes que se presentan a continuación.

\section{Resultados}

A partir del análisis de la información recolectada para describir los elementos del contexto que intervienen en las políticas públicas en los municipios, se identificaron cinco categorías centrales, algunas de ellas con subcategorías: a) políticas y acuerdos municipales; b) eventos que modificaron la SSR; c) actores; d) divulgación; e) implementación; f) contribución social. La figura 1 muestra un mapa conceptual de las categorías elaboradas y la relación entre ellas.

Las políticas públicas en SSR están impulsadas por eventos como hitos, tendencias y cambios conceptuales, de acuerdo con el contexto que se vive en el momento. En su formulación e implementación pueden contribuir conjuntamente actores como el Estado y la sociedad civil, en tanto que su ejecución está ligada a la prestación de los servicios de salud y las estrategias creadas a través de la administración municipal. Esta última establece esas actividades de acuerdo con la normativa establecida a nivel nacional y local. Todo lo anterior contribuye a establecer los logros y aportes sociales que se han alcanzado.

\section{Eventos: hitos y tendencias}

Como resultado de la investigación se encontró que el principal hito social que se ha presentado en el tema de SSR en los últimos años en toda Colombia, y que abarca los municipios en cuestión, es la legalización de la interrupción voluntaria del embarazo (IVE). Sin embargo, según los datos suministrados 


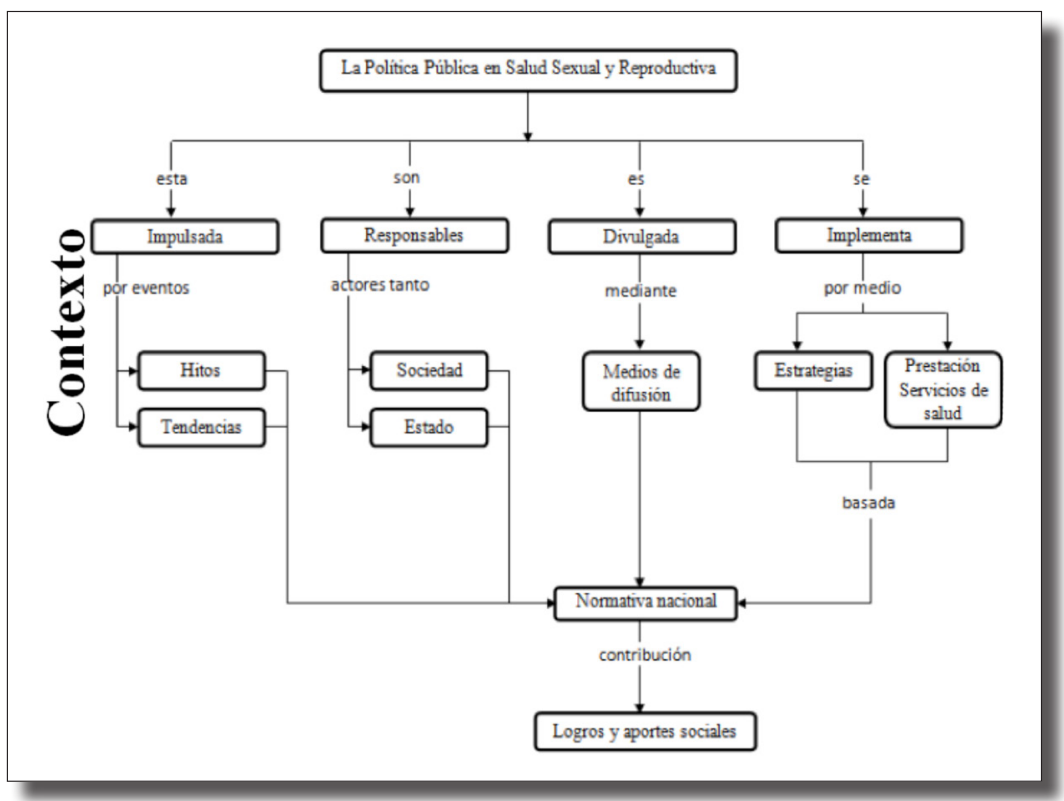

Figura 1.Contexto de LaS POlíticas Públicas EN SSR

Fuente: elaboración propia

por los municipios, dicha práctica está muy poco extendida, pues a la población no se le brinda la información necesaria sobre el tema; sin embargo, se han encontrado avances en algunos municipios, donde se está tratando de incursionar en el tema en las agendas y actividades (13). Según informa uno de los expertos:

[...] por cierto se hacen charlas educativas previstas con ellos individuales, se hacen también pruebas rápidas de VIH, cierto, se canalizan los muchachos a los servicios que ellos tienen derechos ya por la 412 qué es todo lo de promoción y prevención, o sea vayan que ustedes tienen consejerías, cierto, se les da también capacitación a las mujeres sobre la sentencia de la interrupción voluntaria del embarazo. (Entrevista ENT05, enero del 2014)

En Colombia el IVE es un derecho fundamental que tienen las mujeres de cualquier edad. En el 2006 la Corte Constitucional emitió la
Sentencia C-355 y determinó las circunstancias en las cuales las mujeres tienen derecho a abortar: a) cuando el embarazo es producto de una violación sexual o resultado de una inseminación artificial (procedimiento artificial para fecundar un óvulo) y la mujer no dio su consentimiento voluntariamente; b) cuando el embarazo pone en riesgo la vida o la salud de una mujer; c) cuando el feto tiene malformaciones que hacen imposible que viva fuera del útero (13).

En los municipios del Valle de Aburrá donde se trabaja el tema, este se enfoca desde los DSR y es promovido por organismos internacionales como las ONG, la Organización Mundial de la Salud (oms) y el Programa de las Naciones Unidas para el Desarrollo (PNUD), entre otros, con poca difusión y acceso a la información sobre DSR. El Informe de la Cuarta Conferencia Mundial sobre la Mujer, llevada a cabo en Beijing en 1995, reconoció como uno de los DSR de hombres y mujeres “... todas las parejas e individuos 
tienen derecho a decidir libre y responsablemente el número de hijos, el espaciamiento de los nacimientos y el intervalo entre éstos y a disponer de la información y de los medios para ello y el derecho a alcanzar el nivel más elevado de salud sexual y reproductiva" (14).

Otro hito social que tuvo lugar en la ciudad de Medellín fue promovido por el movimiento LGBTI, con la política de diversidad sexual y equidad de género, la cual pretende desarrollar programas dirigidos a la transformación de las situaciones que generan discriminación y exclusión de personas pertenecientes a esta población. En el 2011 este fue uno de los principales temas en los medios de comunicación. Un periódico local publicó: "Medellín se convierte desde hoy en la primera ciudad del país con una política pública para la población LGBT, que le garantiza sus derechos y el libre acceso a todos los servicios del Estado, sin discriminación alguna, al aprobar hoy el Concejo de la ciudad con el respaldo de 16 concejales, un Acuerdo presentado en tal sentido" (15).

En relación con ello surgió el reconocimiento del matrimonio igualitario, donde la Corte Constitucional colombiana, mediante la sentencia C-577 del 2011, estableció que en Colombia sí es constitucional el derecho al matrimonio civil por parte de parejas del mismo sexo, y considera que es el Congreso el que debe regular dicho matrimonio. Usando esta sentencia se podrán realizar matrimonios civiles en cualquier notaría del país, sin necesidad de reglamentación adicional (16).

Estos hitos reflejan un avance significativo en un tema que debate la opinión pública y que ha evidenciado el cambio de enfoque biológico de la SSR referido solo a los aspectos reproductivos, a otros enfoques de tipo social y cultural, así como las nuevas tendencias de la sexualidad: LGBTI, nuevas masculinidades, erotismo, entre otras.

\section{Actores de las políticas públicas: sociedad y Estado}

Gracias a la revisión de la política nacional de SSR se extrajeron los actores que intervienen para dar cumplimiento a dicha política a nivel nacional y municipal, como se muestra en la tabla 3.

En el análisis de la política para el reconocimiento de la diversidad sexual e identidades de género y para la protección, restablecimiento, atención y la garantía de derechos de las personas LGBTI del municipio de Medellín, se encontró que se tiene definida una estructura organizativa con las responsabilidades definidas de la siguiente manera: a) la Secretaría de Cultura Ciudadana ejercerá la coordinación para la orientación, planeación, seguimiento y evaluación; b) el Comité Municipal de Diversidad Sexual e Identidades de Género, bajo su propio reglamento, busca la articulación y coordinación con otros planes y políticas poblacionales. Este comité remplazará a la Mesa de Trabajo LGBTI, que fue creada por Decreto Municipal 1272 del 2007; c) el Consejo Consultivo, conformado por personas u organizaciones que trabajan con personas que se reconocen como LGBTI, y además las representan, es una instancia de la sociedad civil que coopera con la ejecución, el seguimiento y la evaluación de la política.

Por otra parte, la política para la prevención y atención de las violencias sexuales que afectan a la ciudadanía, principalmente a mujeres, niñas y niños en el municipio de Medellín, estableció como responsables de la ejecución y desarrollo de las acciones a las secretarías adscritas a la Alcaldía de Medellín, como la Secretaría de Cultura Ciudadana, la Secretaría de las Mujeres, la Secretaría de Educación, la Secretaría de Salud, la Secretaría de Desarrollo Social, la Secretaría de Gobierno y la Secretaría de Bienestar Social. 
ELEMENTOS DEL CONTEXTO QUE INTERVIENEN EN EL DESARROLLO DE LAS POLITICAS PÚBLICAS DE SALUD SEXUAL Y SALUD REPRODUCTIVA ELABORADAS ENTRE EL 2003 Y EL 2013

Tabla 3. Responsables del cumplimiento de las políticas públicas Según la política nacional de Salud SEXUAL Y REPRODUCTIVA DEL 2003

\begin{tabular}{|l|l|}
\hline Sector salud & Otros sectores \\
Ministerio de la Protección Social & Ministerio de Educación \\
Consejo Nacional de Seguridad Social en Salud & Instituto Colombiano para el Fomento de la \\
Consejos Territoriales de Seguridad Social en Salud & Educación Superior (Icfes) \\
Direcciones Territoriales de Salud & Medios masivos de comunicación \\
Direcciones Locales de Salud & Universidades formadoras de recurso humano \\
Entidades Promotoras de Salud y Administradoras & en salud \\
de Régimen Subsidiado (EPS y ARS) & Instituto Nacional de Medicina Legal y Ciencias \\
Instituciones Prestadoras de Servicios (IPS) & Forenses \\
Instituto Nacional de Salud (INS) & Policía Nacional \\
Instituto Nacional de Vigilancia de Medicamentos y & Ministerio de Comunicaciones \\
Alimentos (Invima) & Fiscalía General de la Nación \\
Instituto Nacional de Cancerología & \\
Instituto Colombiano de Bienestar Familiar (ICBF) & \\
Superintendencia Nacional de Salud & \\
Agencias de cooperación internacional & \\
Organizaciones no gubernamentales & \\
Asociaciones científicas & \\
\hline
\end{tabular}

Fuente: elaboración propia

La revisión de los demás documentos evidencia que en todos los municipios del área metropolitana se hace responsable del tema en SSR a la Secretaría de Salud o de Bienestar Social. Además, se organiza un equipo de salud con profesionales universitarios y asistenciales, pero integrando siempre en el desarrollo de las actividades a otras instituciones pertenecientes al municipio, como son: a) Secretaría de Educación, b) Secretaría de Gobierno, c) Secretaría de Equidad de Género, d) EPS, e) IPS, f) hospital y g) Dirección local de Salud. La figura 2 presenta a los responsables y participantes de las políticas públicas en los municipios analizados.

\section{Medios de difusión}

En las políticas públicas revisadas no se evidencian los medios de difusión o comu- nicación que permitan dar conocimiento a la población de su contenido y ponerlas en el debate público. Asimismo, en las estrategias revisadas, en tres de los municipios no hay medios de difusión, mientras que en los otros seis municipios se encontró que entre sus principales instrumentos de comunicación para la divulgación de las actividades están los medios de comunicación local como boletines, página web, plegables, salvapantallas, adhesivos y cartillas, además de programas radiales, campañas en la zona rural y urbana y reuniones conjuntas con las redes sociales comunitarias.

A este respecto, Martín-Barbero ${ }^{1}$ plantea que los medios de comunicación involucran

1 Jesús Martín-Barbero: doctor en filosofía, estudios de antropología y semiología, experto en cultura y medios de comunicación. 


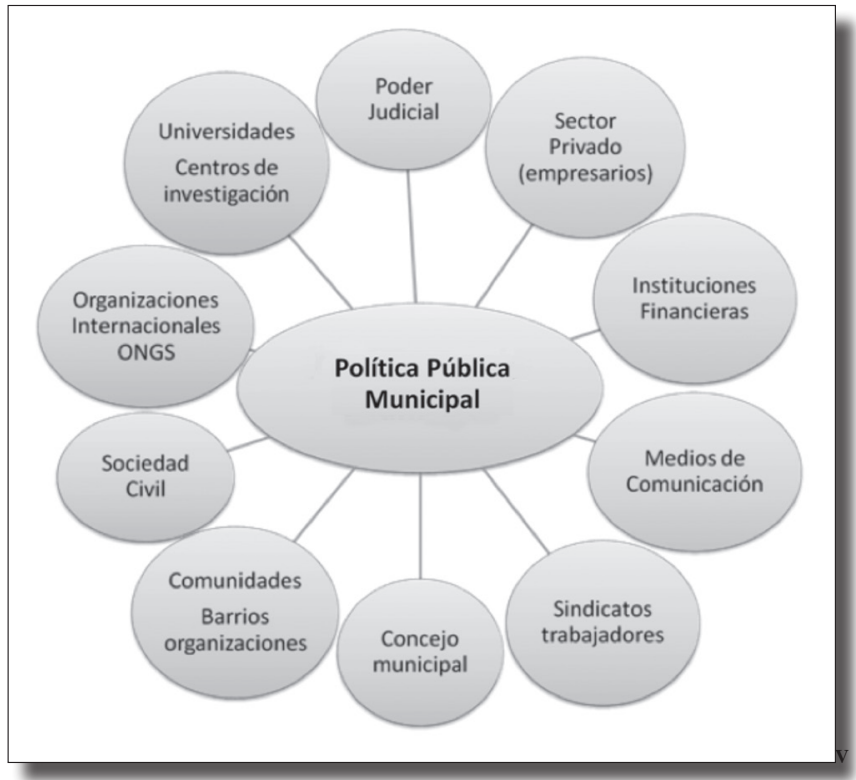

Figura 2. ACtores de las políticas públicas de los municipios

Fuente: elaboración propia

su dimensión como industria, y se debe reconocer sus efectos en las transformaciones de la ciudadanía y la política, la reorganización de la cultura y de las identidades; por lo tanto, es importante plantear la necesidad de pensar en la construcción de las políticas públicas que involucren un subtema de cultura y medios de comunicación con base en el contexto municipal (17).

Han sido varios los académicos comprometidos en abordar el tema e involucrar a los medios de comunicación: periodistas, comunicadores sociales, sociólogos, psicólogos y abogados, entre otros. Esto permite una multiplicidad de voces en los debates sobre el tema de ssR, pues si consideramos que muchos de los documentos escritos son de autoría de políticos, funcionarios de gobierno o empresarios de medios, resulta de gran importancia aquellas posturas que cuestionen, argumenten y descentren la voz oficial con respecto a las políticas (18).
Todo lo anterior demuestra la importante relación entre las políticas públicas y los medios de comunicación, ya que estos desempeñan un papel importante en la comprensión de cualquier tema emitido como política. En este sentido, se encontró que a nivel urbano en los municipios es posible la divulgación del tema en SsR, pero a nivel rural se limita notoriamente la difusión y el entendimiento, pues el contexto hace complicado el acceso de los habitantes rurales a la información.

Implementación de las políticas públicas en SSR

\section{Estrategias de SSR en los planes de desarrollo.} En los documentos enviados por los funcionarios de los municipios se evidenció que solo un municipio tenía establecidas políticas públicas como acuerdo municipal. ${ }^{2}$ Según la entrevista

2 Un acuerdo municipal es un acto jurídico de carácter general. Las atribuciones de orden constitucional las ejercen los concejos. Los acuerdos pueden ser presentados por los concejales o por el 
con uno de los expertos, la administración municipal considera políticas públicas el plan de salud y el plan de desarrollo, por lo cual se decidió realizar un análisis de los planes de desarrollo que, mediante programas, proyectos y estrategias abordan los temas de salud sexual y reproductiva.

Los resultados demuestran que la implementación de las políticas públicas en los municipios del área metropolitana está basada en la Política Nacional de SSR y en los ODM, y se desarrolla por medio de las estrategias de salud incluidas en el plan de desarrollo de cada municipio. Allí se integran las principales metas y actividades, como lo expresa uno de los expertos: “...pero es que las políticas públicas también son el plan de desarrollo, esas son las políticas que nosotros debemos cumplir, un plan municipal de salud con base al plan de desarrollo" (Entrevista ENT06, febrero del 2014).

La política nacional de SSR contempla acciones que confluyen en la promoción de la salud, la prevención y la atención de la enfermedad en el contexto de relaciones adecuadas de costo-efectividad y con el objeto de proteger la salud individual y pública. Las estrategias propuestas para cada uno de las líneas de la política nacional son (3):

\section{- Promoción}

- Coordinación intersectorial e interinstitucional

- Fortalecimiento de la gestión institucional

- Fortalecimiento de la participación

alcalde y, en materias relacionadas con sus atribuciones por los personeros, contralores y las juntas administrativas locales también podrán ser de iniciativa popular, de acuerdo con la ley estatutaria correspondiente.
- Potenciación de las redes sociales de apoyo

\section{- Desarrollo de investigación}

Se encontró que en los municipios, las estrategias desarrolladas están incluidas en los programas y proyectos de los planes de desarrollo de cada territorio, y se evidencia que están integradas en el reporte del Plan Operativo Anual de Inversiones (POAI), herramienta de planificación de la inversión que permite determinar el conjunto de planes, programas y proyectos y la manera como se incorporarán en el presupuesto anual de cada municipio (19). La figura 3 muestra el desarrollo de las políticas públicas en los municipios, se evidencian las estrategias y acciones fundamentadas con base en la Política Nacional de SSR.

Las acciones y estrategias en SSR reflejan el quehacer de los municipios, pero no se ven articuladas a unos objetivos explícitos que hagan efectiva la política y que faciliten la implementación de las estrategias. Aunque las administraciones municipales se encuentran realizando esfuerzos en los temas de ssR, es importante la redacción de objetivos que permitan hacer seguimiento y conocer los resultados, evitando errores en su ejecución o que impidan cumplir con las metas establecidas.

Entre los proyectos más comunes encontrados en los municipios del área metropolitana del Valle de Aburrá están los Servicios de Salud Amigables para Adolescentes y Jóvenes (SSAAJ), el Programa Nacional de Educación para la Sexualidad y Construcción de Ciudadanía y los programas de promoción y prevención del VIH-SIDA, planificación familiar, violencia sexual y enfoque de derechos en la autoconstrucción y manejo de la sexualidad (tabla 4). 


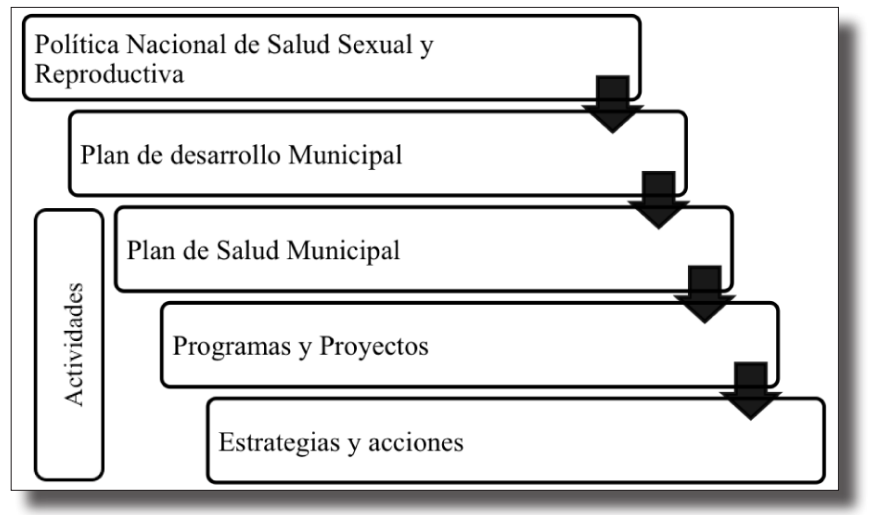

Figura 3. Desarkollo de las políticas públicas en los municipios

Fuente: elaboración propia

En la tabla 4 se evidencia que todos los municipios realizan acciones desde la prevención de la SSR y los factores protectores, mediante actividades educativas desarrolladas, según los documentos, con metodologías participativas y lúdicas, dentro de las que se incluyen talleres, ferias, tomas barriales, charlas, capacitaciones, seminarios, foros y otros materiales.

El análisis de la política pública para el reconocimiento de la diversidad sexual y las identidades de género, y para la protección, el restablecimiento, la atención y la garantía de derechos de las personas LGBTI del municipio de Medellín, sostiene que se adoptan acciones con enfoques de transformación cultural de la sociedad desde los derechos humanos, la orientación sexual y la identidad de género. La política pública para la prevención y atención de las violencias sexuales que afectan a la ciudadanía, principalmente a mujeres, niñas y niños, en el municipio de Medellín, contiene un plan de acción integral, intersectorial, interinstitucional y transdisciplinario, con enfoque diferencial y de género, que contempla acciones de promoción, prevención, detección, atención, sanción, rehabilitación, vigilancia y control, de acuerdo con las particularidades y especificidades de los sectores poblacionales.
Prestación de servicios de salud en SSR. La prestación de los SSR en Colombia incluye las actividades, procedimientos e intervenciones contemplados en el Plan Nacional de Salud Pública ${ }^{3}$ y en el Plan Obligatorio de Salud (POS) ${ }^{4}$ como servicios individuales ambulatorios y hospitalarios, donde se hace educación, promoción, prevención y atención de infecciones de transmisión sexual, control prenatal y perinatal, partos, abortos, atención a cáncer de órganos reproductivos, atención a los casos de violencia sexual, consultas para jóvenes, consultas de ginecología, urología y actividades extramurales (20).

Por otra parte, en la Resolución 412 del 2006 se establecen las actividades, procedimientos e intervenciones de demanda inducida y obligatorio cumplimiento, y se adoptan las normas técnicas para el desarrollo de las

3 El Plan Nacional de Salud Pública reúne un conjunto de actividades, intervenciones y procedimientos de promoción de la salud, prevención de la enfermedad, vigilancia en salud pública y control de factores de riesgo, dirigidos a la colectividad de manera gratuita, y no está sujeto a ningún tipo de afiliación en salud.

4 Plan Obligatorio de Salud: conjunto de servicios de atención en salud a los que tiene derecho un usuario, cuya finalidad es la protección de la salud, la prevención y curación de enfermedades. Incluye el suministro de medicamentos para el afiliado y su grupo familiar, junto al reconocimiento de indemnizaciones en caso de incapacidad por enfermedad y maternidad. 
ELEMENTOS DEL CONTEXTO QUE INTERVIENEN EN EL DESARROLLO DE LAS POLÍTICAS PÚBLICAS DE SALUD SEXUAL Y SALUD REPRODUCTIVA ELABORADAS ENTRE EL 2003 Y EL 2013

TABLA 4. ACCIONES Y ACTIVIDADES DE LOS MUNICIPIOS 2012-2015

\begin{tabular}{|c|c|c|}
\hline Municipio & Acciones & Actividades \\
\hline Barbosa & $\begin{array}{l}\text { Programa de promoción y prevención } \\
\text { en sSR a mujeres en edad fértil y ado- } \\
\text { lescentes } \\
\text { Servicios amigables para adolescentes } \\
\text { y jóvenes }\end{array}$ & $\begin{array}{l}\text { Cinco programas radiales } \\
\text { Cien encuentros educativos } \\
\text { Veinticinco campañas de citología en zona } \\
\text { urbana y rural } \\
\text { Seis reuniones de la Red Social de Apoyo }\end{array}$ \\
\hline Bello & $\begin{array}{l}\text { Programa de SSR con enfoque de } \\
\text { derecho } \\
\text { Servicios amigables para adolescentes } \\
\text { y jóvenes } \\
\text { Plan de protección integral a la niñez }\end{array}$ & $\begin{array}{l}\text { Tomas barriales dos veces al mes con progra- } \\
\text { mas de promoción y prevención sSR } \\
\text { Charlas y talleres ITS, VIH, métodos de planifi- } \\
\text { cación familiar, embarazo adolescente, abuso } \\
\text { sexual, resiliencia y habilidades para la vida } \\
\text { Boletines mensuales en derechos sexuales y } \\
\text { reproductivos }\end{array}$ \\
\hline Copacabana & --- & $\begin{array}{l}\text { Implementación de la estrategia nacional de } \\
\text { prevención de embarazo en adolescentes } \\
\text { Aumento casos de abuso detectados }\end{array}$ \\
\hline Envigado & $\begin{array}{l}\text { Programa Proyectos Educativos Ins- } \\
\text { titucionales (PEI), educación sexual y } \\
\text { reproductiva } \\
\text { Programa de capacitación en Salud } \\
\text { Sexual y Reproductiva } \\
\text { Campañas del Programa de Educación } \\
\text { para la Sexualidad y Construcción de } \\
\text { Ciudadanía (PEscc) del Ministerio de } \\
\text { Educación Nacional } \\
\text { Campaña Abre tus ojos, prevención } \\
\text { de explotación, reclutamiento, mal- } \\
\text { trato y abuso sexual de niños, niñas y } \\
\text { adolescentes } \\
\text { Escuela de Formación y Participación } \\
\text { Ciudadana } \\
\text { Módulo de diversidad sexual e identi- } \\
\text { dad de género con enfoque de derecho }\end{array}$ & $\begin{array}{l}\text { Nueve ejes transversales incorporados a los } \\
\text { Proyectos Educativos Institucionales (PEI) } \\
55 \text { establecimientos educativos públicos in- } \\
\text { tervenidos } \\
88 \text { capacitaciones } \\
95 \text { actividades educativas } \\
\text { Tres campañas } \\
\text { Un módulo de diversidades sexuales e identi- } \\
\text { dades de géneros creado } \\
\text { Cuatro acciones educativas implementadas } \\
\text { de LGBTI } \\
\text { Ocho talleres pedagógicos para familias y } \\
\text { comunidad en general realizados }\end{array}$ \\
\hline Girardota & $\begin{array}{l}\text { Campañas del Programa de Educación } \\
\text { para la Sexualidad y Construcción de } \\
\text { Ciudadanía (PESCC) del Ministerio de } \\
\text { Educación Nacional } \\
\text { Feria de Salud Sexual y Reproductiva }\end{array}$ & $\begin{array}{l}\text { Una brigada de APS en tema de planificación } \\
\text { familiar, control prenatal y toma de citología } \\
\text { Charlas informativas SSR } \\
\text { Seis encuentros intergeneracionales de SSR } \\
\text { Campañas informativas de factores protecto- } \\
\text { res en salud sexual }\end{array}$ \\
\hline
\end{tabular}




\begin{tabular}{|c|c|c|}
\hline & Acciones & Actividades \\
\hline La Estrella & $\begin{array}{l}\text { Servicios amigables para adolescentes } \\
\text { y jóvenes } \\
\text { Campañas del Programa de Educación } \\
\text { para la Sexualidad y Construcción de } \\
\text { Ciudadanía (PESCC) del Ministerio de } \\
\text { Educación Nacional } \\
\text { Talleres de derechos sexuales y re- } \\
\text { productivos, planificación familiar y } \\
\text { enfermedades de transmisión sexual }\end{array}$ & $\begin{array}{l}\text { Diez talleres a grupos gestantes de la impor- } \\
\text { tancia del control prenatal } \\
1500 \text { plegables sobre métodos anticonceptivos } \\
10 \% \text { de incremento de personas ingresadas } \\
\text { a planificación } \\
\text { Cuatro estrategias de educación para la sexua- } \\
\text { lidad y construcción de ciudadanía } \\
\text { Seminarios y foros para docentes y padres } \\
\text { de familia }\end{array}$ \\
\hline Medellín & $\begin{array}{l}\text { Programa Jóvenes por la Vida } \\
\text { Programa Unidades Móviles de Salud } \\
\text { Programa dispensador de condones } \\
\text { Prevención de violencias sexuales } \\
\text { Escuelas y colegios saludables }\end{array}$ & $\begin{array}{l}\text { Quinientos dispensadores instalados } \\
4000 \text { material físico } \\
190 \text { actividades de información y comunica- } \\
\text { ción ssR } \\
117632 \text { preservativos entregados }\end{array}$ \\
\hline Rionegro & $\begin{array}{l}\text { Programa de educación y promoción } \\
\text { de factores protectores de SSR } \\
\text { Comité Técnico Territorial en SSR } \\
\text { Servicios amigables para adolescentes } \\
\text { y jóvenes } \\
\text { Campaña asesoríay prueba voluntaria VIH }\end{array}$ & $\begin{array}{l}\text { Cuatro actividades de información } \\
\text { Diez convocatorias para trabajar el tema SSR } \\
\text { Unidad de servicios amigable operando } \\
\text { Doce programas de formación }\end{array}$ \\
\hline Sabaneta & $\begin{array}{l}\text { Programa Educación como medio para } \\
\text { promocionar la salud } \\
\text { Programa Autoconstrucción y manejo } \\
\text { de la sexualidad } \\
\text { Programa de planificación familiar } \\
\text { mujeres en edad escolar secundaria }\end{array}$ & $\begin{array}{l}\text { Treinta talleres sSR } \\
\text { Cincuenta consultas psicológicas } \\
1100 \text { preservativos entregados } \\
\text { Sesenta anticonceptivos entregados } \\
\text { Sesenta personas ingresadas a planificación }\end{array}$ \\
\hline
\end{tabular}

Fuente: elaboración propia

acciones de protección específica y detección temprana, que se encuentran muy ligadas a los temas de la Política Nacional de sSR. Los temas más relevantes en SSR son (21):

- Atención al parto

- Atención en planificación familiar para hombres y mujeres

- Detección temprana de las alteraciones del desarrollo del joven (10-29 años)
- Detección temprana de las alteraciones del embarazo

- Detección temprana del cáncer de cuello uterino

- Detección temprana del cáncer de seno Igualmente, en la prestación del servicio en SSR se definen las guías de atención de enfermedades de interés en salud pública (21): 
- Enfermedades de transmisión sexual (infección gonocóccica, sífilis, VIH)

- Hipertensión arterial y hemorragias asociadas al embarazo

\section{- Menor y mujer maltratados}

- Lesiones preneoplásicas del cuello uterino

En los municipios, las secretarías de salud deben garantizar la prestación del servicio según el municipio de residencia de la población, así como la totalidad de las actividades, procedimientos e intervenciones en SSR, a través de la red prestadora de los servicios de salud. Esta situación se hace compleja, por la filiación y el acceso a la prestación de los servicios en los temas de SSR (22).

Según los datos encontrados, en los municipios del área metropolitana se ha visto afectada la calidad de la prestación de los servicios en SSR, pues existen acciones de duplicidad en el desarrollo de las estrategias establecidas, ya que las secretarías no están integradas con acciones compartidas; adicionalmente, no se le da continuidad al servicio, por la fragmentación en la atención. Sumado a lo anterior, existe una economía de mercado en el sector salud que se preocupa solo por los ingresos y la capacidad de pago de la población o del Estado, a lo cual un experto argumenta: “... está el factor, digamos económico, que ha distorsionado radicalmente la red de prestación de servicios, y donde cada vez son menos los que prestan servicios [...] Yo no creo que haga falta presupuesto, yo lo que creo es que el presupuesto se despilfarra contratando mil contratistas, cada uno hacer una cosita distinta" (Entrevista ENT01, septiembre del 2013).

Los resultados muestran que la mayoría de las acciones que los municipios realizan en los temas de SSR están dirigidas a actividades de promoción y prevención, según el Plan Nacional de Salud Pública, pues dicho presupuesto está a cargo del sistema de salud colombiano y la gobernación de Antioquia, en el caso de los municipios.

A este respecto, la Organización Panamericana de la Salud (ops) sostiene:

La fragmentación de los servicios de salud es una causa importante del bajo desempeño de los servicios de salud y por lo tanto del pobre rendimiento general de los sistemas de salud. Las dificultades de acceso a los servicios que enfrenta la población, los servicios de pobre calidad técnica, el uso irracional e ineficiente de los recursos, la baja satisfacción de los usuarios, son algunas de las consecuencias que genera la fragmentación por si misma o en conjunto con otros factores. (23)

Normativa. Después de la recolección de las políticas públicas en los municipios sobre temas en ssR, solo se encontró que en Medellín existen dos documentos considerados normativos como políticas públicas, emitidos por el Concejo, lo cual demuestra que los demás municipios formulan las estrategias y acciones con base en la Política Nacional de SSR.

Los planes de desarrollo de los municipios contienen las actividades que se ejecutan para el mejoramiento de la salud sexual y reproductiva de la comunidad, para lo cual se basan en las políticas que existen a nivel nacional. Estos planes se consideran como un instrumento rector de la planeación municipal y por ello beben de fuentes normativas y de las políticas públicas, según los temas que establezca la administración municipal, con el objeto de asegurar el uso eficiente de sus recursos y el desempeño adecuado de sus funciones, además de darle la orientación a los programas que se formulen y que 
respondan a los compromisos adquiridos en el programa de gobierno municipal.

\section{Contribución: logros y aportes sociales}

Esta investigación intenta aportar evidencia sobre los logros y aportes sociales que han dejado a la comunidad los elementos que intervienen en el contenido estructural de las políticas públicas en salud sexual y reproductiva.

El logro más relevante que se encontró dentro del análisis de las políticas sobre SSR a nivel nacional y municipal fue la gran atención que ha recibido el tema en la sociedad y los diferentes medios. De allí han partido las acciones y estrategias que han desarrollado los municipios dentro de sus políticas y planes de desarrollo, permitiendo trabajar las líneas de acción en documentos como el Plan Decenal de Salud. En este sentido, uno de los expertos comenta: “... para el área metropolitana podríamos decir tal vez que se posicionó el tema de la salud sexual y reproductiva como un tema dentro de la opinión pública [...] creo que básicamente el logro más importante es posicionamiento del tema" (Entrevista ENT03, diciembre del 2013).

Otro experto nos dice: “... vamos con base al programa de salud sexual y reproductiva publicado por la gobernación de Antioquia creo que fue en el año 2006 [...] todo ese tiempo con base en estos lineamientos pero vamos a cambiarlo conforme al Plan decenal de ahora" (Entrevista ENT06, febrero del 2014).

Otro aporte que se pudo encontrar en la política para el reconocimiento de la diversidad sexual y las identidades de género, que busca la protección, el restablecimiento, la atención y la garantía de los derechos de las personas LGBTI del municipio de Medellín, fue que 26 permitió fomentar el trabajo activo de los activistas de la comunidad LGTBI en los otros municipios, así como otros movimientos de diferentes sectores de la sociedad. Adicionalmente, dejó para la ciudad el Centro de Diversidad Sexual, espacio que busca reconocer e impulsar los procesos organizativos, la creatividad artística, cultural y académica y las propuestas de este segmento de la población, como lo argumentó un experto en el tema:

[...] el sitio se creó con el fin de reconocer a la LGTBI como una población visible, que merece ser escuchada, reconocida y que necesita espacios como estos para lograr incubar procesos que le muestren a la ciudad por qué Medellín tiene que empezar a pensarse como una ciudad diversa [...] trabajar en la transformación de imaginarios culturales en torno a este grupo, fomentar y fortalecer los procesos organizativos, culturales y participativos de la población. (24)

Además, el análisis evidenció que la política pública para la prevención y atención de las violencias sexuales que afectan a la ciudadanía, principalmente a mujeres, niñas, niños y adolescentes en el municipio de Medellín, trajo como aporte a la ciudad el reconocimiento del problema como una manifestación de violencia creciente. Adicionalmente, en el año 2012 se publicó un boletín del Observatorio de Violencias Sexuales en la ciudad como mecanismo de comunicación y difusión, pretendiendo visibilizar el problema de la violencia sexual y aportar a las soluciones.

\section{Discusión}

En Colombia el cumplimiento del IVE se ha visto vulnerado por la objeción de conciencia de algunos médicos, como expresión de una postura ética o religiosa para realizar el procedimiento, y por la suspensión provisional que desde el 2009 dictaminó el Consejo de 
Estado cuando declaró la nulidad del Decreto 4444 del 2006, que reglamentó la prestación del servicio. La entidad en mención argumentó que la expedición del decreto por parte del presidente de la República desbordó sus competencias, pues debió estar precedido por una ley que lo reglamentara. Con esta decisión el Gobierno y el Consejo Nacional de Seguridad Social tendrán que reglamentar la IVE a través de otros mecanismos (25).

El incumplimiento de este derecho ejemplifica que la formulación de las políticas públicas no garantiza su cumplimiento, hace falta la implementación y el empoderamiento de los actores para cumplir con esos derechos, donde muchas veces es necesario la utilización de otros instrumentos legales como la tutela (26).

En cuanto al hito, el matrimonio igualitario, existen dos posturas divididas: la de la Iglesia y el procurador, quienes están en contra, y la del movimiento LGTBI y algunos jueces que consideran el tema como prioritario por definir. El debate sigue, por lo cual las distintas agremiaciones adoptan posturas propias a favor de sus asociados. La discusión sigue abierta y con alta grado de polémica, porque el Congreso no ha reglamentado el tema (27).

Un elemento importante es reconocer a los responsables de las políticas públicas durante el diseño, formulación e implementación, esto evidenciará la movilización de los actores, con quiénes se podrá contar, quiénes podrán ser los más interesados, quiénes saldrán beneficiados, quiénes podrían verse afectados, quiénes serán indiferentes, quiénes podrán aportar y de qué manera y quiénes podrán oponerse.

Otro elemento que se pudo encontrar con la revisión de los documentos y de los discursos de los expertos es que los medios de comunicación, periodistas, investigadores y analistas de políticas, desempeñan un papel importante en la divulgación de las políticas públicas en salud sexual y reproductiva en la sociedad, pudiendo distorsionar la información o contribuyendo de forma crítica al tema, fomentando el cambio hacia nuevas actitudes, el respeto por los derechos humanos, las responsabilidades ciudadanas y el compromiso político para impulsar los temas en SSR.

La implementación de las políticas públicas en SSR en los municipios, mediante estrategias y acciones producto de los programas y proyectos en salud sexual que se encuentran establecidos en el plan de salud municipal, la realizan las secretarías de salud. Estos programas y proyectos a su vez son extraídos de los planes de desarrollo municipales elaborados por los alcaldes y deben fundamentar el tema con base en la política nacional del 2003.

El elemento encontrado como común denominador en las estrategias utilizadas por los municipios son los programas de educación y los servicios amigables para adolescentes y jóvenes, los cuales son impulsados por el Ministerio de Educación. Los hallazgos señalan que las políticas públicas propias en SSR para los municipios son emitidas por el Concejo como acuerdos, pero una estrategia puede ser impulsada también como política por la sociedad civil, sin que exista un documento público. Sin embargo, es importante que dicha estrategia sea reglamentada para que se establezca como guía para los funcionarios y demás actores, y que además permita establecer y evidenciar los logros y aportes en su implementación.

Los resultados sugieren que la política nacional de ssR fue creada como pauta para los entes departamentales y municipales, pero estos deben adaptar los lineamientos que propone la política a las necesidades 
propias de la población según las regiones, permitiendo mejorar la prestación del servicio según el contexto de sus habitantes.

Por tanto, los hallazgos demuestran que los documentos analizados han hecho algún aporte a la comunidad, aunque no siempre se pueda continuar con las estrategias planteadas. Esto evidencia que a pesar de que los documentos no se encuentren estructurados con todos los componentes establecidos como políticas, lo desarrollado hasta el momento ha contribuido al bienestar de la comunidad.

\section{Conclusiones}

Los municipios no tienen políticas públicas propias en ssR desarrolladas, sin embargo, procuran adaptar la política nacional de ssR bajo el esquema de proyectos en el plan de desarrollo municipal. Esto evidencia que para desarrollar políticas es necesario elaborar proyectos que a su vez estén alineados con los programas, los cuales tienen componentes de SSR.

Las dos políticas públicas locales sobre el tema de ssR se localizaron solo en el municipio de Medellín, lo que evidencia que falta fortalecer la gestión administrativa en los demás municipios locales y darle importancia no solo a nivel salud-enfermedad, sino también a nivel integral, pues la salud sexual y reproductiva afecta los determinantes sociales de la población.

No se encontraron investigaciones a nivel local anteriores a este estudio que aborden las políticas públicas en SSR y que pudieran servir de insumo para brindar alternativas de solución y toma de decisiones que incidan en la formulación e implementación de políticas de salud sexual y reproductiva para la población de cada uno de los municipios. Estos tienen condiciones distintas y, en esa medida, requieren políticas públicas particulares según sus necesidades.
Tanto el Estado como la administración municipal son los responsables de la formulación e implementación de las políticas públicas, no solo en salud sexual y reproductiva, sino también en todos los temas que involucran a la población. Además, es cierto que se hace necesario la participación de la sociedad en el diseño, la gestión y la evaluación de las políticas y que no solo sea vista como destinataria.

La participación de los diferentes sectores de la sociedad en la construcción de políticas públicas en SSR es aún muy incipiente, una de las razones podría ser la poca difusión del Estado y de los diversos medios de comunicación en cuanto a la formulación e implementación de dichas políticas dirigidas a la comunidad.

El logro más significativo de SSR en los municipios es la puesta de los temas en la palestra pública, a nivel municipal, lo que ha permitido que ciertos grupos de la población trabajen los contenidos considerados tabús para la sociedad, como la diversidad sexual, aportando un inicio para el desarrollo de actividades desde las necesidades y condiciones de la población de cada municipio.

\section{Agradecimientos}

Esta publicación es resultado de investigación realizada con el apoyo financiero de la Estrategia de sostenibilidad 2013-2014 del Comité para el Desarrollo de la Investigación (CODI) Universidad de Antioquia

\section{Referencias bibliográficas}

1. Maoño C, Vásquez N. Derechos sexuales y reproductivos [Internet] [acceso: 15 de abril del 2015]. Disponible en: http://www.dicc.hegoa.ehu.es/listar/ mostrar/66.

2. Organización de las Naciones Unidas. Informe de la Conferencia Internacional sobre la Población y 
el Desarrollo. El Cairo, 5 a 13 de septiembre de 1994 [Internet] Nueva York: onU; 1995 [acceso: 31 de marzo del 2015]. Disponible en: https://www. unfpa.org/webdav/site/global/shared/documents/ publications/2004/icpd_spa.pdf.

3. Organización de las Naciones Unidas. Declaración del Milenio [Internet]. Nueva York: ONU; 2000 [acceso: 31 de marzo del 2015]. Disponible en: http:// www.un.org/spanish/milenio/ares552.pdf.

4. Pantelides E, Bott S. Reproducción, salud y sexualidad en América Latina. Buenos Aires: Biblos-OMs; 2000.

5. Salazar C. Las políticas públicas: actualizada y complementada. Bogotá: Fundación Cultural Javeriana de Artes Gráfica; 1999.

6. Colombia, Ministerio de la Protección Social. Política Nacional de Salud Sexual y Reproductiva [Internet]. Bogotá: Ministerio de la Protección Social; 2003 [acceso: 27 de julio del 2013]. Disponible en: http://www.hsph.harvard.edu/population/ domesticviolence/colombia.reprohealth.03.pdf.

7. Área Metropolitana del Valle de Aburrá. Indicadores de impacto [Internet]. [acceso: 27 de julio del 2013]. Disponible en: http://www.areadigital.gov. co/institucional/Paginas/IndicadoresImpacto.aspx

8. United Nations Fund for Population Activities (UNFPA). Estado de la población mundial 2011 [Internet] [acceso: 27 de julio del 2013]. Disponible en: http://foweb.unfpa.org/swP2011/reports/ SP-SwOP2011_Final.pdf.

9. Organización Mundial de Salud. Políticas públicas para la salud pública [Internet] [acceso: $27 \mathrm{de}$ julio del 2013]. Disponible en: http://www.who.int/ whr/2008/chapter4/es/index.html.

10. Roth AN. Análisis de las políticas públicas: de la perspectiva basada en el anarquismo epistemológico. Ciencia Política [Revista en internet] 2007 [acceso: 20 de abril del 2015]. 3: 6-29. Disponible en: http://www.revistas.unal.edu.co/index.php/ cienciapol/article/view/17522/18379.

11. Yuni J. Técnicas para investigar: recursos metodológicos para la preparación de proyectos de investigación. Argentina: Editorial Brujas; 2006.

12. Colecciones electrónicas [Internet]. Medellín: Universidad de Antioquia; 2013 [acceso: 27 de mayo del 2013]. Disponible en: http://www.udea. edu.co/portal/page/portal/SistemaDeBibliotecas.

13. Corte Constitucional colombiana. Sentencia C-355, Interrupción voluntaria del embarazo. Bogotá: Corte Constitucional; 2006.

14. Informe de la Cuarta Conferencia Mundial sobre la Mujer. Beijing 1995 Sep. 4-15. Beijing: Naciones Unidas; 1995.

15. Medellín es la primera ciudad en el país con política pública para el sector LGBT. Caracol Noticias.com 2011, abril 16; Sec. Noticias Regionales [Internet] [acceso: 18 de febrero del 2014]. Disponible en: http://www.caracol.com.co/noticias/regionales/ medellin-es-la-primera-ciudad-en-el-pais-conpolitica-publica-para-el-sector-lgbt/20110416/ nota/1456385.aspx.

16. Corte Constitucional colombiana. Sentencia C-577. Corte declaró la exequibilidad de artículo 113 del
Código Civil que define el matrimonio civil en Colombia y exhortó al Congreso de la República a legislar de manera sistemática y organizada sobre los derechos de las parejas del mismo sexo, con el objeto de eliminar el déficit de protección existente. Bogotá: Corte Constitucional; 2011.

17. Martín-Barbero J. De los medios a las mediaciones: Comunicación, cultura y hegemonía. Segunda edición. Barcelona: Editorial Gustavo Gili; 1991.

18. Roth A-N. Perspectivas teóricas para el análisis de las políticas públicas: ¿de la razón científica al arte retórico? Estudios Políticos, Instituto de Estudios Políticos. Medellín: Universidad de Antioquia; 2008, pp. 67-91.

19. Consejo Nacional de Política Económica y Social República de Colombia Departamento Nacional de Planeación. Conpes 3753: Plan Operativo Anual de Inversión. 2013. Bogotá: Conpes; 2013.

20. Colombia, Congreso de la República. Ley 1122 de 2007 por la cual se hacen algunas modificaciones en el Sistema General de Seguridad Social en Salud y se dictan otras disposiciones. Diario Oficial 46506. Bogotá: El Congreso; 2007.

21. Colombia, Ministerio de Salud. Resolución 412 del 2000, febrero 25, por la cual se establecen las actividades, procedimientos e intervenciones de demanda inducida y obligatorio cumplimiento y se adoptan las normas técnicas y guías de atención para el desarrollo de las acciones de protección específica y detección temprana y la atención de enfermedades de interés en salud pública. Bogotá: El Ministerio; 1993.

22. Colombia. Ministerio de la Protección Social. Decreto 4444 del 2006 por el cual se reglamenta la prestación de unos servicios de salud sexual y reproductiva. Diario Oficial 46481. Bogotá: El Ministerio; 2006.

23. Organización Panamericana de Salud. Redes integradas de servicios de salud. [Internet] [acceso: 17 de marzo del 2014]. Disponible en: http://www. paho.org/hq/index.php?option $=$ com_content $\&$ vie $\mathrm{w}=$ article \&id $=4305$ :redes-integradas-de-serviciosde-salud\&Itemid $=3553 \&$ lang $=$ es.

24. Medellín Cultural. Medellín abre su sede de la diversidad sexual [Internet] [acceso: 17 de marzo del 2014]. Disponible en: http://www.medellincultura. gov.co/ciudadania/Paginas/C_centrodeladiversidadsexualydegenero.aspx.

25. Colombia, Ministerio de la Protección Social. Decreto 4444 del 2006: por el cual se reglamenta la prestación de unos servicios de salud sexual y reproductiva. Diario Oficial 46481. Bogotá: Corte Constitucional; 2006.

26. Colombia. El Presidente. Decreto 2591 de 1991 por el cual se reglamenta la acción de tutela consagrada en el artículo 86 de la Constitución Política. Diario Oficial 40165. Bogotá: Corte Constitucional; 1991.

27. El Espectador. Notarios retan al procurador por matrimonio igualitario. Elespectador.com 2013, junio 21; Sec. Política [Internet] [acceso: 18 de febrero del 2014]. Disponible en: http://www.elespectador. com/noticias/politica/notarios-retan-al-procuradormatrimonio-igualitario-articulo-420449. 